Monatsschr Kinderheilkd 2019 • 167:34-35 https://doi.org/10.1007/s00112-018-0546-7 Online publiziert: 20. Juli 2018

(c) Der/die Autor(en) 2018

CrossMark

\author{
A. Berger ${ }^{1,2,3} \cdot$ U. Kiechl-Kohlendorfer ${ }^{1,4} \cdot$ J. Berger ${ }^{1,2,5} \cdot$ A. Dilch $^{1,2,6} \cdot$ M. Kletecka- \\ Pulker $^{7} \cdot$ B. Urlesberger ${ }^{1,8} \cdot$ M. Wald ${ }^{1,9} \cdot$ M. Weissensteiner ${ }^{1,10} \cdot$ H. Salzer ${ }^{1,2,11}$ \\ ${ }^{1}$ Arbeitsgruppe für Neonatologie und Pädiatrische Intensivmedizin, Österreichische Gesellschaft für \\ Kinder- und Jugendheilkunde (ÖGKJ), \\ http://www.docs4you.at/Content.Node/OEGKJ/AGs/neonatologie_und_intensivmedizin.php \\ ${ }^{2}$ Arbeitsgruppe für Ethik in der Kinder- und Jugendheilkunde, Österreichische Gesellschaft für Kinder- \\ und Jugendheilkunde (ÖGKJ), http://www.docs4you.at/Content.Node/OEGKJ/AGs/ethik.php \\ ${ }^{3}$ Klinische Abteilung für Neonatologie, Pädiatrische Intensivmedizin und Neuropädiatrie, \\ Universitätsklinik für Kinder- und Jugendheilkunde, Medizinische Universität Wien, Wien, Österreich \\ ${ }^{4}$ Department für Kinder- und Jugendheilkunde, Univ.-Klinik für Pädiatrie II (Neonatologie), Medizinische \\ Universität Innsbruck, Innsbruck, Österreich \\ ${ }^{5}$ Abteilung für Kinder- und Jugendheilkunde, Wilhelminenspital der Stadt Wien, Wien, Österreich \\ ${ }^{6}$ Abteilung für Kinder- und Jugendheilkunde, SMZ Süd der Stadt Wien, Wien, Österreich \\ ${ }^{7}$ Institut für Ethik und Recht in der Medizin, Universität Wien, Wien, Österreich \\ ${ }^{8}$ Klinische Abteilung für Neonatologie, Univ.-Klinik für Kinder- und Jugendheilkunde, Medizinische \\ Universität Graz, Graz, Österreich \\ ${ }^{9}$ Division für Neonatologie, Universitätsklinik für Kinder- und Jugendheilkunde, Paracelsus Medizinische \\ Privatuniversität, Salzburg, Österreich \\ ${ }^{10}$ Klinik für Neonatologie, Med. Campus IV, Kepler Universitätsklinikum, Linz, Österreich \\ "Klinische Abteilung für Kinder- und Jugendheilkunde, Universitätsklinikum Tulln, Tulln, Österreich
}

\title{
Geburtsmodus bei extremer Frühgeburtlichkeit
}

\section{Änderungen an der Leitlinie zur Erstversorgung von Frühgeborenen an der Grenze der Lebensfähigkeit}

Nach Erscheinen der Leitlinie zur Erstversorgung von Frühgeborenen an der Grenze der Lebensfähigkeit [1] gab es Diskussion mit Vertretern der Österreichischen Gesellschaft für Geburtshilfe und Gynäkologie (ÖGGG) bezüglich der Passage zum optimalen Geburtsmodus an der Grenze der Lebensfähigkeit. Während vonseiten der Neonatologie an der Grenze der Lebensfähigkeit (Schwangerschaftswoche [SSW] 23, SSW 24) auf Basis der vorhandenen Literatur ein Vorteil durch eine Sectio-Entbindung gesehen wird, wird diese Literatur vonseiten der Geburtshilfe dahingehend interpretiert, dass keine Empfehlung für einen bevorzugten Geburtsmodus ausgegeben werden kann. In einer gemeinsamen Besprechung zwischen ÖGGG und Arbeitsgruppe (AG) Neonatologie/ Pädiatrische Intensivmedizin der Österreichischen Gesellschaft für Kinder- und Jugendheilkunde (ÖGKJ) gab es eine Einigung, die eine Änderung des bestehenden Textes der Leitlinie vorsieht, auf dass die vorhandene Leitlinie auch vonseiten der ÖGGG konsentiert wird.

Konkret wird von den Autoren der Leitlinie folgende Modifikation des LLTextes vorgesehen:

Statt: Die vorhandene Datenlage legt nahe, dass bei extremer Frühgeburtlichkeit eine von erfahrenen GeburtshelferInnen ausgeführte Kaiserschnittentbindung auch aus Schädellage einen Vorteil bezüglich Mortalität und Morbidität des Kindes bringt. Auch lässt sich belegen, dass Frühgeburten zu den Tagesrandzeiten sowie an Sonn- und Feiertagsdiensten, v. a. aber nachts, mit einem schlechteren Outcome verbunden sind als Frühgeburten während der Kernar- beitszeit. Bei mit großer Wahrscheinlichkeit absehbarer Frühgeburt an der Grenze der Lebensfähigkeit erscheint deshalb eine „geplante“ Kaiserschnittgeburt für das Kind von Vorteil.

NEU: Zum optimalen Entbindungsmodus an der Grenze der Lebensfähigkeit liegen naturgemäß kaum randomisierte Daten vor, und die Ergebnisse der publizierten Studien sind widersprüchlich. Einzelne Studien scheinen einen Vorteil in Bezug auf Mortalität und Hirnblutungsrate durch Kaiserschnittentbindung nahezulegen; andere finden keinen Unterschied. Letztendlich muss der optimale Entbindungsmodus individuell festgelegt werden.

Die aktualisierte Fassung der Leitlinie findet sich unter https://doi.org/10.1007/ s00112-018-0532-0. 


\begin{tabular}{l}
\hline \\
\hline Korrespondenzadresse \\
\hline Prof. Dr. A. Berger \\
Klinische Abteilung für Neonatologie, \\
Pädiatrische Intensivmedizin und Neuro- \\
pädiatrie, Universitätsklinik für Kinder- und \\
Jugendheilkunde, Medizinische Universität \\
Wien \\
Währinger Gürtel 18-20, 1090 Wien, Österreich \\
angelika.berger@meduniwien.ac.at \\
\hline
\end{tabular}

Funding. Open access funding provided by Medical University of Vienna.

\section{Einhaltung ethischer Richtlinien}

Interessenkonflikt. A. Berger, U. Kiechl-Kohlendorfer, J. Berger, A. Dilch, M. Kletecka-Pulker, B. Urlesberger, M. Wald, M. Weissensteiner und H. Salzer geben an, dass kein Interessenkonflikt besteht.

Dieser Beitrag beinhaltet keine von den Autoren durchgeführten Studien an Menschen oder Tieren.

Open Access. Dieser Artikel wird unter der Creative Commons Namensnennung 4.0 International Lizenz (http://creativecommons.org/licenses/by/4.0/deed. de) veröffentlicht, welche die Nutzung, Vervielfältigung, Bearbeitung, Verbreitung und Wiedergabe in jeglichem Medium und Format erlaubt, sofern Sie den/die ursprünglichen Autor(en) und die Quelle ordnungsgemäßnennen, einen Linkzur Creative Commons Lizenz beifügen und angeben, ob Änderungen vorgenommen wurden.

\section{Literatur}

1. Berger A, Kiechl-Kohlendorfer U, Berger J et al (2017) Erstversorgung von Frühgeborenen an der Grenze der Lebensfähigkeit. Monatsschr Kinderheilkd 165(2):139-147. https://doi.org/10. 1007/s00112-016-0149-0 (Online publiziert: 16. August 2016)

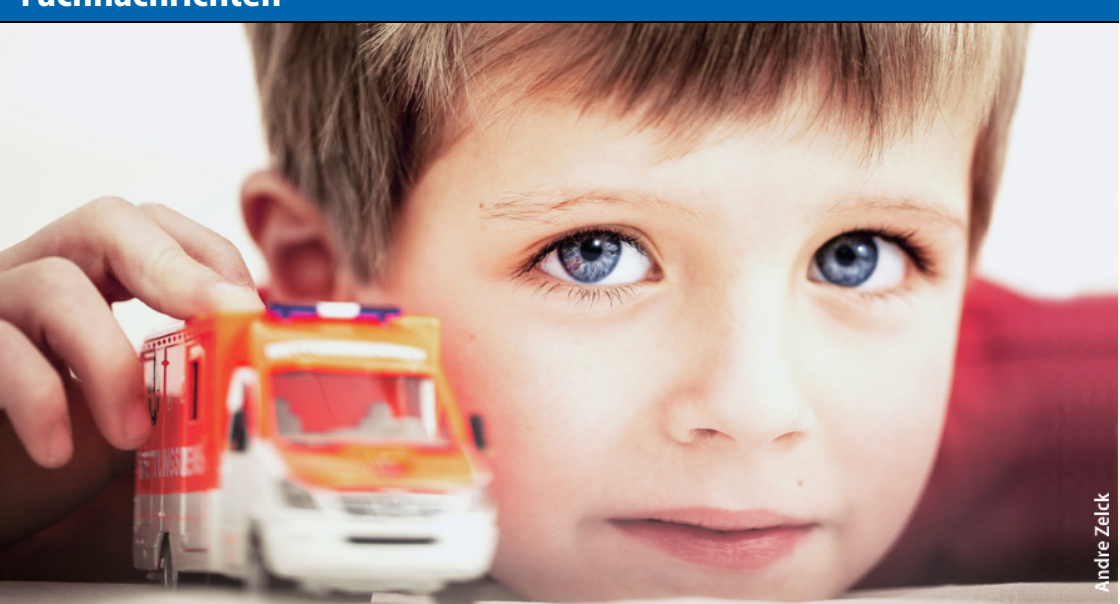

\section{Deutscher Forschungspreis für Kinderheilkunde ausgeschrieben}

Hermann-Seippel-Preis, dotiert mit 200.000 Euro, für Prävention in der Pädiatrie

Zum zweiten Mal lobt die Stiftung Universitätsmedizin den „Hermann-SeippelPreis - Deutscher Forschungspreis für Kinderheilkunde“ unter der Schirmherrschaft von Dr. Eckhart von Hirschhausen aus. Der Fokus liegt in diesem Jahr auf dem Gebiet der gesundheitlichen Prävention bei Kindern und Jugendlichen. Bewerbungen können bis zum 31.03.2019 eingereicht werden.

Wer kann sich bewerben?

Bewerben können sich ForscherInnen sowie Forschergruppen medizinischer Fakultäten in ganz Deutschland, die ihr Forschungsvorhaben im Bereich Kinderheilkunde und im Rahmen eines Kooperationsprojekts mit der Medizinischen Fakultät der Universität Duisburg-Essen umsetzen und erfolgreich weiterentwickeln möchten. Die Stiftung Universitätsmedizin Essen, die das Preisgeld bereitstellt, kann satzungsgemäß unmittelbar nur Vorhaben auf dem Gebiet der Universitätsmedizin Essen fördern. Daher muss auch ein Beteiligter des Forschungsvorhabens die Universitätsmedizin Essen vertreten. Für VertreterInnen der Universitätsmedizin Essen ist eine Bewerbung nur in Kooperation mit Vertreterlnnen einer anderen deutschen medizinischen Fakultät möglich.

Welche Projektideen können eingereicht werden?

Die eingereichten Forschungsvorhaben sollen einen klaren Bezug zur Verbesserung der Präventionsmedizin im Bereich der Kinderheilkunde besitzen und ein hohes Potenzial wissenschaftlicher Innovation vorweisen. Übergreifendes Ziel ist es, Erkrankungen von Kindern und Jugendlichen vorzubeugen (Primärprävention), frühzeitig zu erkennen (Sekundärprävention) und ein Fortschreiten der Krankheit sowie Folge- und Begleiterkran- kungen zu verhindern (Tertiärprävention), um zur Verbesserung der Kindergesundheit beizutragen.

Das Preisgeld kann sowohl für Sach- und Investitionsmittel als auch für personelle Ressourcen verwendet werden.

Wie kann ich mich bewerben? Die Bewerbung erfolgt mithilfe eines Antragsformulars, verfügbar zum Download unter www.forschungspreis-kinderheilkunde.de. Bewerbungen sowie ein kurzer Lebenslauf können bis zum 31. März 2019 eingereicht werden.

\section{Hintergrund}

Die Hermann-Seippel-Unterstützungseinrichtung $\mathrm{GmbH}$ war ein Tochterunternehmen der E.ON Ruhrgas AG bzw. ihres Rechtsnachfolgers. Satzungsgemäß kommt der Liquidationserlös gemeinnützigen Zwecken zugute. Die Stiftung Universitätsmedizin Essen erhielt 1 Mio. Euro, um damit einen Forschungspreis für Kinderheilkunde auszuloben.

Nähere Informationen Jorit Ness, Geschäftsführer Stiftung Universitätsmedizin Essen, Tel.: 0201-7234699, jorit.ness@uk-essen.de

Quelle: Stiftung Universitätsmedizin Essen 